BULL. AUSTRAL. MATH. SOC.

$76 \mathrm{BI}, 76 \mathrm{~B} 25$

VOL. $30(1984), 315-316$

\title{
WAVE PROPAGATION IN DENSITY STRATIFIED FLUIDS
}

\author{
JOHn ANTHONy GEAR
}

This thesis falls into three distinct sections. In Chapter one the fundamental properties of internal gravity waves are investigated, with respect to the effect that the basic physical quantities of fluid velocity, and buoyancy frequency, have upon the propagation of these waves. Among other things, it is shown that when the wavenumber tends to infinity the wave phase speeds are then found to depend only upon the local behavior of the mean flow near an overall maximum or minimum of the velocity profile. Also, the phase speed and group velocity are plotted against wavenumber, when the fluid velocity has a hyperbolic tangent and a hyperbolic secant profile. Several interesting phenomena are observed in these plots.

In shallow stratified fluids internal solitary waves are described, to first order in wave amplitude, by the Korteweg-de Vries equation; the solution for a single solitary wave has the familiar "sech" -profile and a phase speed which varies linearly with wave amplitude. In Chapter Two this theory is extended to second order in wave amplitude. The second order correction to the wave profile and the phase speed and the first order correction to the wavelength are all determined. Four special cases are considered in detail. It is shown that in certain special circumstances the first order theory may fail due to the vanishing of the nonlinear coefficient in the Korteweg-de Vries equation. When this occurs it is shown that a different theory is required which leads to an equation with both quadratic and cubic nonlinearities.

Received 18 June 1984. Thesis submitted to University of Melbourne, January 1984. Degree approved June 1984. Supervisor: Dr R. Grimshaw.

Copyright Clearance Centre, Inc. Serial-fee code: 0004-9727/84 $\$ A 2.00+0.00$. 
In Chapter Three the strong interaction between weakly nonlinear long internal gravity wave modes is studied. Strong interactions occur when the wave phase speeds are nearly equal although the waves belong to different modes. In shallow stratified fluids it is shown that this situation is described by two coupled Korteweg-de Vries equations, which possess both dispersive and nonlinear coupling terms. It is shown that these coupled equations possess three conserved quantities as well as an exact analytical solution involving the characteristic "sech" Vries equation. Also it is shown that when the coefficients satisfy some special conditions, the coupled equations possess an n-solution solution in terms of the Korteweg-de Vries n-solution solution. In general though the coupled equations are found not to be amenable to solution by inverse scattering technique, and thus a numerical method has been employed in order to solve the equations. This numerical method is described in detail in Appendix A. Finally several numerical solutions of the coupled equations are presented.

Aeronautical Research Laboratories,

PO Box 4331,

Melbourne,

Victoria 3001,

Australia. 